\title{
Effectiveness and toxicity of lenvatinib in refractory thyroid cancer: Dutch real-life data
}

\author{
M D Aydemirli', E Kapiteijn', K R M Ferrier², P B Ottevanger ${ }^{3}$, T P Links ${ }^{4}$, A N A van der Horst-Schrivers ${ }^{4}$, \\ K E Broekman', R H H Groenwold ${ }^{6}$ and J Zwaveling7
}

${ }^{1}$ Department of Medical Oncology, Leiden University Medical Centre, Leiden, The Netherlands, ${ }^{2}$ Department of Pharmacology, ${ }^{3}$ Department of Medical Oncology, Radboud University Medical Centre, Nijmegen, The Netherlands, ${ }^{4}$ Department of Endocrinology, ${ }^{5}$ Department of Medical Oncology, University Medical Centre Groningen, Groningen, The Netherlands, ${ }^{6}$ Department of Clinical Epidemiology, and ${ }^{7}$ Department of Clinical Pharmacology and Toxicology, Leiden University Medical Centre, Leiden, The Netherlands

Correspondence should be addressed to J Zwaveling

Email

J.Zwaveling@lumc.nl

\section{Abstract}

Objective: The SELECT trial showed progression-free survival (PFS) benefit for lenvatinib for advanced radioiodinerefractory differentiated thyroid cancer (RAI-refractory or RR-DTC) patients, on which current clinical practice is based. We assessed whether the effectiveness and toxicity of lenvatinib in real-life clinical practice in the Netherlands were comparable to the pivotal SELECT trial.

Methods: From three Dutch centres Electronic Health Records (EHRs) of patients treated in the lenvatinib compassionate use program or as standard of care were reviewed and checked for SELECT eligibility criteria. Baseline characteristics, safety, and efficacy measures were compared and PFS and overall survival (OS) were calculated.

Furthermore, PFS was compared to estimates of PFS reported in other studies.

Results: A total of 39 DTC patients with a median age of 62 years were analysed. Of these, 27 patients (69\%) did not fulfil the SELECT eligibility criteria. The most common grade $\geq 3$ toxicities were hypertension $(n=11,28 \%)$, diarrhoea $(n=7,18 \%)$, vomiting $(n=4,10 \%)$, and gallbladder disease $(n=3,8 \%)$. Median PFS and median OS were $9.7(95 \%$ confidence interval (Cl): 4.0-15.5) and 18.3 (95\% Cl: 4.9-31.7) months, respectively, response rate was 38\% (95\% Cl: 23-54\%). PFS in the Dutch real-life situation was comparable to previous real-life studies, but inferior to PFS as shown in the SELECT trial $(P=0.04)$.

Conclusions: PFS in our non-trial population was significantly shorter than in the SELECT trial population. In the interpretation of results, differences in the real-life population and the SELECT study population regarding patient characteristics should be taken into account.

\section{Introduction}

Differentiated thyroid cancer (DTC) accounts for the majority $(\sim 95 \%)$ of thyroid cancer $(1,2)$, including the main histologic subtypes of papillary (PTC), follicular (FTC) thyroid cancer and Hürthle cell carcinoma. With standard therapy, including total thyroidectomy with subsequent radioactive iodine (RAI) and thyrotropin suppression therapy, the majority of DTC is cured (3). However, a $5-15 \%$ of patients develops metastatic or recurrent disease, a part of whom (26-60\%) progresses to RAI-refractory DTC (RR-DTC) $(4,5,6)$. RR-DTC forms the major source of TC-related deaths with less than $10 \%$ 10-year survival $(5,7)$. Currently two tyrosine kinase inhibitors (TKIs), sorafenib and lenvatinib, are approved for RR-DTC and several other TKIs have been studied in trials (8). Lenvatinib (E7080) is a multi-targeted TKI, of VEGFR 1-3, FGFR 1-4, PDGFR $\alpha$, RET, KIT, which are involved in tumour growth and maintenance (9), which has shown efficacy in progressive RR-DTC.

Published by Bioscientifica Ltd. 
The randomized phase 3 SELECT trial showed a significant increase in median progression-free survival (PFS) of 14.7 months compared to placebo in RR-DTC patients with a response rate of $64.8 \%$ and disease-control rate of $87.7 \%$ (7). A recent updated analysis showed a median PFS of 19.4 months in the lenvatinib-treated group versus 3.7 months in the placebo group and among the lenvatinib-treated patients, 33.1 months in responders (responders defined as patients having demonstrated complete response (CR) or partial response (PR) as best overall response (BOR), according to Response Evaluation Criteria in Solid Tumours (RECIST) v1.1), and 7.9 months in non-responders (10).

Clinical trials provide the evidence base for medicine, yet, are generally performed under strict conditions. For instance, in the SELECT trial, only patients with minimal comorbidity and up to only one prior TKI therapy regimen were allowed to enter the study. Therefore, real-life (observational) data of a non-trial population may extend the knowledge about the effectiveness of lenvatinib in clinical practice and may bridge the gap between stringently controlled and less controlled, more heterogenous patient population in clinical practice. Therefore, baseline characteristics, efficacy and toxicity measures of non-trial lenvatinib DTC recipients were collected from three tertiary care centres for the treatment of RAI-refractory thyroid cancer patients in the Netherlands. These data were evaluated for effectiveness and toxicity, and compared with other real-life studies.

\section{Subjects and methods}

\section{Patients}

All consecutive DTC patients treated with lenvatinib for RR-DTC from March 2015 to January 2019 at the Leiden University Medical Centre (LUMC), University Medical Centre Groningen (UMCG) and Radboud University Medical Centre (RadboudUMC), which are tertiary care centres in the Netherlands for advanced thyroid cancer, were evaluated. Follow-up and treatment were according to local practice. Efficacy measures included response evaluation performed according to RECISTv1.1 by computed tomography (CT) imaging (reported as: CR, complete response; $\mathrm{PR}$, partial response; $\mathrm{SD}$, stable disease; PD, progressive disease) circa every 3 months, clinical assessment and in indicated cases ${ }^{18} \mathrm{~F}$-fluorodeoxyglucose positron emission tomography ( ${ }^{18} \mathrm{~F}$-FDG-PET)-CT and/or magnetic resonance imaging (MRI). The present study was reviewed by the Medical Ethics Review Committee of the LUMC, Leiden, of the UMCG, Groningen and of the Radboudumc, Nijmegen, and determined that the Medical Research Involving Human Subjects Act (WMO) did not apply to this study.

\section{Lenvatinib treatment}

Based on the SELECT study, the recommended initial dose of lenvatinib was $24 \mathrm{mg}$ once daily. Optionally preceded by dose interruptions up to 28 days for grade 2 and higher adverse events, sequential reduced doses were 20, 14, 10 mg once daily, or an alternative $18 \mathrm{mg}$ reduced dose. In case of intolerability or toxicity, the onset of comorbidity or a clinical course apt for potential severe toxic interaction, a metabolic remission, a request of exemption by the patient, the lenvatinib treatment was interrupted or ceased. In the case of disease progression, lenvatinib treatment was ceased as well, with few exceptional cases in which treatment was prolonged due to clinical benefit.

\section{Patient characteristics and outcome}

Baseline characteristics of the lenvatinib-treated patients included in the study were compared to those of the patients included in the SELECT study and checked for the SELECT eligibility criteria. Median PFS, median OS, disease-control rate (DCR), response rate (RR) and $\mathrm{BOR}$ were analysed as efficacy outcomes. OS was defined as the time of initiation of lenvatinib therapy to death or last day of follow-up and PFS as the time from initiation of lenvatinib therapy to PD, death, or last day of follow-up. The RR denotes the proportion of patients with a CR or PR as BOR. The DCR designates the proportion of patients with a $\mathrm{CR}$ or $\mathrm{PR}$ or $\mathrm{SD}$ as BOR. Adverse events were retrospectively retrieved from patient electronic health records (EHRs) and toxicity was, if possible, graded according to Common Terminology Criteria for Adverse Events (CTCAE) version 4.0.

\section{Statistical analysis}

Data were summarised as mean with ranges (minimummaximum) or as counts with percentages. Median PFS and median OS were estimated using the Kaplan-Meier method. Based on the data presented in the paper by Schlumberger et al. (7), the Kaplan-Meier curve of the PFS in the SELECT study was simulated and used as a reference for comparison to our real-life data using a $\log$ rank (Mantel-Cox) test. A $P$ value of $<0.05$ was 
considered statistically significant. Statistical analyses were performed using SPSS statistical software (IBM SPSS Statistics for Windows, version 23 (IBM Corp.)). A forest plot of median PFS was created based on reported median PFS and 95\% CIs from the real-life studies by Berdelou et al. (11), Balmelli et al. (12), Nervo et al. (13), the current study, and the clinical trials by Schlumberger et al. (7) and Sugino et al. (14). For the study by Berdelou et al. (11), we used the information displayed in figure 1 of that publication to obtain estimates of the median PFS. The 95\% confidence interval of the median PFS was estimated using the method described by Simon (15).

\section{Results}

\section{Study population}

Clinical records of 39 patients with advanced differentiated thyroid cancer treated with lenvatinib were analysed. Lenvatinib treatment was initiated after a median of 5 years (range: 0-19) from initial thyroid cancer diagnosis. Thirty-six (92\%) patients were initially treated according to current standard of care including total thyroidectomy, RAI ablation therapy and TSH suppression therapy. Of all patients, $20(51 \%)$ were men, median age was 62 years (range: 43-80), histologic subtype was PTC in 10 (26\%), follicular variant of PTC (FV-PTC) in 5 (13\%), FTC in 9 (23\%), Hürthle cell carcinoma in 15 (39\%). Baseline characteristics of the patients are listed in Table 1.

Compared to the patients included in the SELECT study, the age and sex of our real-life population were similar. Regarding histologic subtype, in our population, Hürthle cell carcinoma comprised the majority of cases (39 vs $18.4 \%$ in SELECT), the share of follicular thyroid carcinoma was similar (23 vs $20.3 \%$ in SELECT), of papillary thyroid carcinoma was less (39 vs $50.6 \%$ ). The percentage of patients with bone metastases (74 vs 39.8\% in SELECT) and patients who received prior TKI therapy (77 vs $25.3 \%$ in SELECT) was higher in our population. A somewhat higher percentage of patients in our non-trial population had an ECOG 2 or 3 compared to the SELECT population (16 vs $5 \%$ ). The majority of our non-trial population $(n=27,69 \%)$ did not fulfil the eligibility criteria for the SELECT trial. Reasons for exclusion would have been more than one prior TKI treatment $(n=9,23 \%)$, lack of thyroidectomy/RAI therapy $(n=3,8 \%)$. Other reasons included another malignancy within a year of initiation of lenvatinib treatment $(n=3,8 \%)$. Furthermore, significant comorbidity was observed in 8 patients (21\%) (gastric
Table 1 Baseline characteristics of real-life lenvatinib-treated patients.

\begin{tabular}{lc}
\hline Characteristic & All patients $(n=39)$ \\
\hline Age, years (median, range) & $62(43-80)$ \\
Sex, $n$ (\%) & $19(49)$ \\
Female & $20(51)$ \\
Male & \\
Performance status, $n$ (\%) & $15(39)$ \\
ECOG 0 & $18(46)$ \\
ECOG 1 & $5(13)$ \\
ECOG 2 & $1(3)$ \\
ECOG 3 & \\
Histologic type, $n(\%)$ & $10(26)$ \\
Papillary & $5(13)$ \\
Papillary, FV & $9(23)$ \\
Follicular & $15(39)$ \\
Hürthle cell & $30(77)$ \\
Prior TKI therapy, $n$ (\%) & $9(23)$ \\
No & $21(54)$ \\
1 TKI & $4(10)$ \\
2 TKIs & $5(13)$ \\
3 TKIs & $34(87)$ \\
Previous treatment*, $n(\%)$ & $17(43)$ \\
Surgery/RFA/REmb & $29(73)$ \\
Radiation & $12(31)$ \\
Combination & $29(74)$ \\
Bone metastasis, $n$ (\%) & $73(43-153)$ \\
Weight, kg (median, range) & $0.06(0-17)$ \\
TSH, mU/L (median, range) & $22.5(6.1-100)$ \\
FT4, pmol/L (median, range) & \\
\hline & \\
\hline
\end{tabular}

*After initial thyroidectomy and radioactive iodine therapy, if applicable. ECOG, Eastern Cooperative Oncology Group performance status; FV, follicular variant; REmb, radio-embolization; RFA, radio frequent ablation; TKI, tyrosine kinase inhibitor.

bypass, partial paraplegia, IgA nephropathy, colectomy, immune thrombocythemia; morbus Crohn, atrial fibrillation) that would have prohibited participation in the SELECT trial. Furthermore, hypertension before start of treatment with lenvatinib $>150 / 90 \mathrm{mmHg}(n=6,15 \%)$ and TSH levels $>0.50 \mathrm{mIU} / \mathrm{L}(n=5,13 \%)$, also exclusion criteria for the SELECT trial, were observed in our patients.

\section{Treatment course}

In our population the median duration of lenvatinib use was 6.1 months (range 0.1-35.9) and mean dosage was 18.6 mg per day. Thirty-three patients (85\%) started treatment at a daily dosage of $24 \mathrm{mg}, 2$ (5\%) started at $20 \mathrm{mg}, 2(5 \%)$ started at $18 \mathrm{mg}, 2$ (5\%) started at $14 \mathrm{mg}$ (Fig. 1). Dose reductions were applied in more than half of our patients: $22(56 \%)$. Dosages were reduced during treatment to 20 $\mathrm{mg}$ (11 patients, $28 \%$ ), $14 \mathrm{mg}$ (20 patients, $51 \%$ ) or $10 \mathrm{mg}$ $(n=5,13 \%)$. Dose interruptions were applied in 25 patients (64\%). Thirty-six interruptions were due to toxicity $(n=21$, 


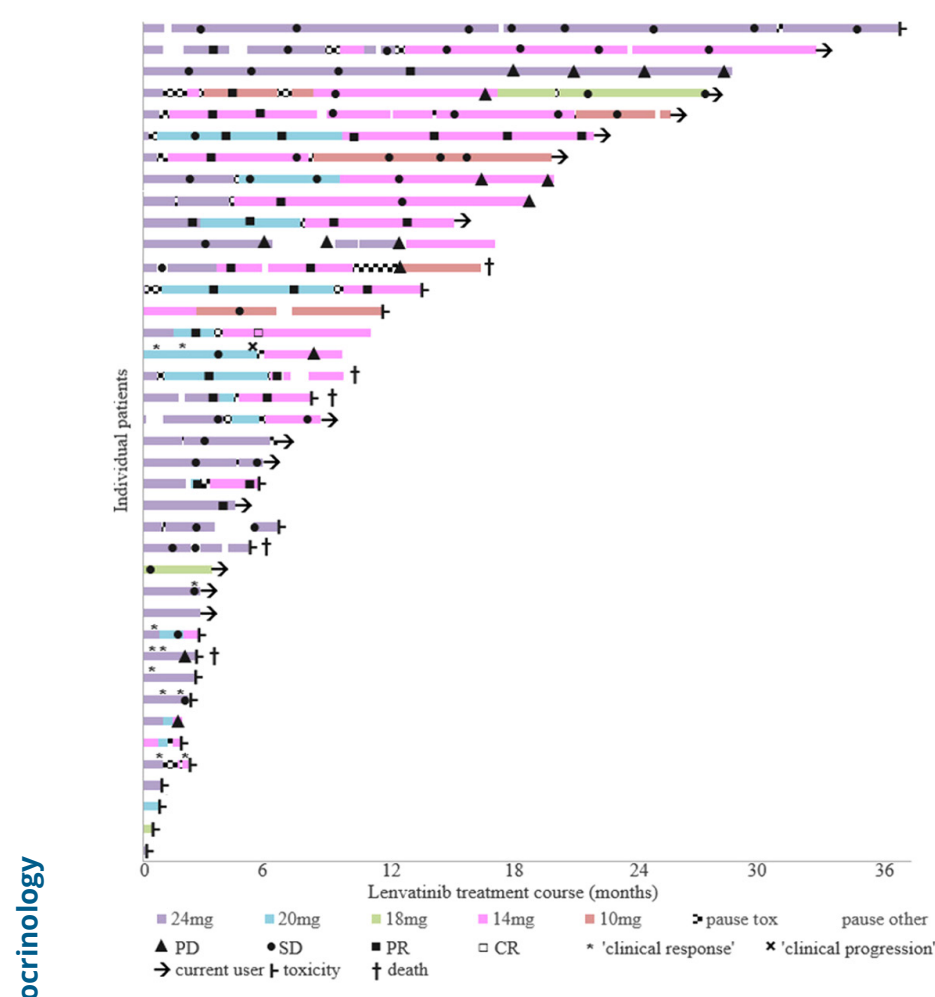

\section{Figure 1}

Lenvatinib therapy course in 39 real-life RR-DTC patients. Depicted in colour bars, the use of lenvatinib for various doses $(24,20,18,14,10 \mathrm{mg})$ through time (days, x-axis) per patient (y-axis). Drug toxicity led to dose reduction in indicated cases. Pauses due to lenvatinib toxicity are depicted by a check pattern. Temporary stops due to other causes are unmarked. Current use is indicated $(\rightarrow)$, toxicity as a reason for ceasing lenvatinib $(\vdash)$, death during treatment $(t)$, or if toxicity induced death is not ruled out $(1+)$. Additional objective measures of response evaluation according to RECIST ( $\triangle P D, \triangle S D, \square P R$, $\square$ CR) are indicated through time. Few subjective, clinically observed signs appraised as response $\left({ }^{*}\right)$ or progression $(x)$ are indicated additionally; these include altered swollenness of lymph nodes and subcutaneous metastases evaluated on physical examination. $\mathrm{CR}$, complete response; $\mathrm{PD}$, progressive disease; PR, partial response; SD, stable disease.

$54 \%)$ and 23 interruptions ( $n=12,31 \%)$ were due to other causes including radiotherapy and surgery (Fig. 1).

Twenty-six patients (67\%) had discontinued treatment at the time of analysis, due to therapy-related toxicity $(n=15,39 \%)$, PD $(n=5,13 \%)$, death $(n=5,13 \%)$, including three patients for whom treatment-emerged adverse effects were not excluded and for one patient due to CR. PD was noted in nine patients during treatment of whom six patients continued use due to clinical benefit.

\section{Drug toxicity}

Treatment-related adverse events were common and are listed in Table 2. The most common adverse events (any grade) were asthenia, hypertension, decreased weight, diarrhoea, nausea and decreased appetite. The most common serious (grade $\geq 3$ ) toxicities included hypertension $(n=11,28 \%)$, diarrhoea $(n=7,18 \%)$ and vomiting $(n=4,10 \%)$. Serious AEs that could not be excluded to be lenvatinib-related, occurred in 7 patients (18\%). In general, hypertension could be managed with antihypertensive agents, but in two cases, treatment with lenvatinib was interrupted. Symptoms of palmarplantar dysesthesia syndrome (PPED), oral dysesthesia and stomatitis could be managed by topical treatment for relief of symptoms in most cases, but necessitated interruption of lenvatinib treatment in three patients. Decreased appetite and decreased weight were managed by dietary measures. Most common adverse events leading to dose reduction and interruptions included combinations of decreased weight, loss of appetite, asthenia, diarrhoea and nausea.

Twenty-two patients (56\%) were hospitalized during lenvatinib treatment. 12 patients (31\%) were hospitalized likely due to lenvatinib-related toxicity. This included a combination of asthenia, diarrhoea, nausea, (impending) dehydration, insufficient intake in six patients (15\%), one of whom also had recurrent severe hypocalcaemia. Furthermore, gastro-intestinal perforation in four patients (10\%), due to which one patient died, and sepsis leading to death due to colitis in one patient, although widely disseminated disease was not ruled out. Gall bladder stones/cholecystitis in three patients (8\%), heart failure in two patients (5\%) and hypertension in one patient (3\%) led to hospitalisation of lenvantinib-treated patients.

Other reasons for hospitalisation were intermittent (palliative local) radiotherapy (five patients, 13\%) and other advanced disease-related complications including fever (three patient, 8\%), dyspnoea (one patient, 3\%) and local palliative surgery (three patients, $8 \%$ ).

One patient (3\%) experienced cerebellar infarction; a vascular effect of lenvatinib was considered possible/ not excluded. One patient (3\%) developed a thyrotoxic crisis in 2 days after lenvatinib initiation leading to death shortly after ceasing lenvatinib; differential diagnostically it was thought to be related to a pathologic fracture with incompliant use of thyroxine and not likely to be related to lenvatinib.

Including the aforementioned patient with sepsis due to colitis and the patient with intestinal perforation, five patients died while on treatment. Other causes of death 
Table 2 Treatment-related adverse events (AEs) in real-life lenvatinib-treated patients.

\begin{tabular}{|c|c|c|}
\hline \multirow[b]{2}{*}{ Adverse event } & \multicolumn{2}{|c|}{ All patients $(n=39) \boldsymbol{n}(\%)$} \\
\hline & Any grade & Grade $\geq 3$ \\
\hline Any AEs & $39(100)$ & $21(54)$ \\
\hline Most common AEs & 37 (95) & $15(38)$ \\
\hline Asthenia & $25(64)$ & $3(8)$ \\
\hline Hypertension & $25(64)$ & $11(28)$ \\
\hline Decreased weight & $20(51)$ & \\
\hline Diarrhoea & $22(56)$ & $7(18)$ \\
\hline Nausea & $19(49)$ & $2(5)$ \\
\hline Decreased appetite & $15(384)$ & $1(3)$ \\
\hline TSH changes & $14(36)$ & \\
\hline PPED & $10(26)$ & \\
\hline Myalgia & $12(31)$ & \\
\hline Vomiting & $10(26)$ & $4(10)$ \\
\hline Arthralgia & $10(26)$ & \\
\hline Dysphonia & $8(21)$ & \\
\hline Oral dysesthesia & $7(18)$ & \\
\hline Headache & $6(15)$ & \\
\hline Dysgeusia & $4(10)$ & \\
\hline Dry mouth & $3(8)$ & \\
\hline Oropharyngeal pain & $4(10)$ & \\
\hline Hypocalcaemia & $4(10)$ & $1(3)$ \\
\hline Stomatitis & $2(5)$ & \\
\hline Thrombocytopenia & $3(8)$ & \\
\hline Alopecia & $3(8)$ & \\
\hline Obstipation & $3(8)$ & \\
\hline $\begin{array}{l}\text { Serious AEs, treatment } \\
\text { relatedness not excluded }\end{array}$ & $9(23)$ & $7(18)$ \\
\hline Gastro-intestinal perforation & $4(10)$ & $2(5)$ \\
\hline Gallbladder disease & $3(8)$ & $3(8)$ \\
\hline Heart failure & $2(5)$ & $2(5)$ \\
\hline Colitis & $1(3)$ & $1(3)$ \\
\hline $\begin{array}{l}\text { Serious AEs, probably not } \\
\text { treatment related }\end{array}$ & $2(5)$ & $2(5)$ \\
\hline Thyrotoxicosis & $1(3)$ & $1(3)$ \\
\hline Cerebellar infarction & $1(3)$ & $1(3)$ \\
\hline
\end{tabular}

PPED, palmar-plantar dysesthesia syndrome.

included progression of disease $(n=2,5 \%)$, heart failure $(n=1,3 \%)$. Death occurred in 12 patients after treatment when lenvatinib was stopped, including the patient who developed a thyrotoxic crisis shortly after initiating lenvatinib; 22 patients were still alive at the time of data extraction.

\section{Efficacy}

Median PFS was 9.7 months (95\% CI: 4.0-15.5; Fig. 2) and median OS was 18.3 months (95\% CI: 4.9-31.7; Fig. 3). One patient had a CR (4.0 months), 14 patients a PR (33\%; 6.6 months, range: 3.0-23.6), 14 patients SD (37\%; 2.8 months, range: $0.1-8.4), 2$ patients PD (7\%; 1.9 months, range: $0.5-2.0)$ as best overall response. The BOR was not evaluable in 8 patients (21\%). The response rate was 38\%

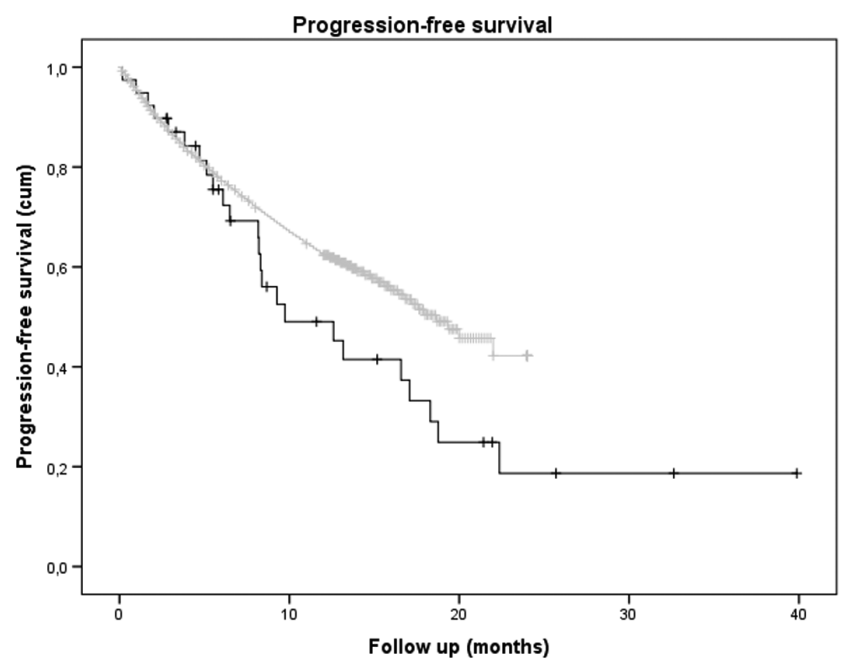

Figure 2

Kaplan-Meier curves of progression-free survival of 39 real-life lenvatinib-treated DTC patients (black curve) and simulated data based on the SELECT study (grey curve). KM curves plotted for comparison, log rank test (Mantel-Cox), $P=0.04$.

(95\% CI: 23-54\%) and disease control rate was 74\% (95\% CI: 61-88\%) (Table 3).

Compared to the SELECT study the median PFS in our real-life patients was significantly shorter (log rank (Mantel-Cox), $P=0.04)$, Fig. 2. The response rate was lower in our population (38 vs $64.8 \%$ in SELECT), as well as the disease control rate (74 vs $87.7 \%$ ). More of our patients had SD (37 vs $23.0 \%$ ), less had PR (33 vs 63.2\%) and more of our cases were not evaluable (21 vs $5.4 \%$ ).

Our data regarding PFS were comparable to other studies featuring the outcome of lenvatinib in real life (11, 12, 13): median PFS ranged from 6 to 12 months. The PFS outcomes from all the available real-life data for lenvatinib, along with the results reported for two randomised trials (7, 14), have been visualised in a forest plot (Fig. 4).

\section{Discussion}

In the present study, efficacy and toxicity of lenvatinib in the treatment of RR-DTC patients in the Netherlands were assessed in real-life daily clinical practice. The disease control rate of $74 \%$ and median PFS of 9.7 months (95\% CI: 4.0, 15.5) confirm efficacy of treatment with lenvatinib in advanced RR-DTC in clinical practice, but PFS in our study was significantly shorter than in the phase 3 SELECT trial. Furthermore, toxicity often resulted in drug interruptions or dose reductions. 


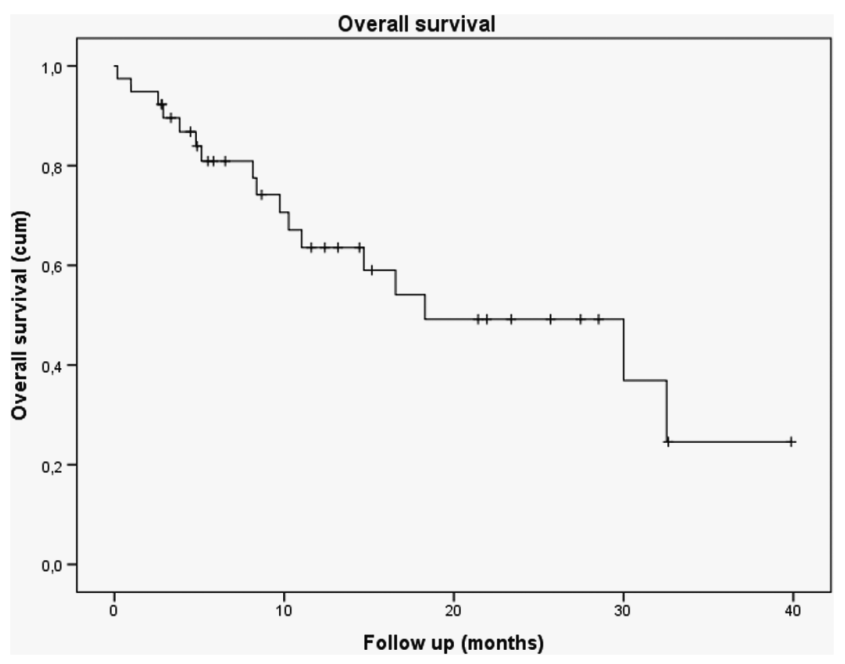

Figure 3

Kaplan-Meier curves of overall survival in 39 real-life lenvatinib-treated DTC patients.

Half of our non-trial population did not tolerate lenvatinib at the initial dosage, but only at a reduced dose. Not only the dose reductions, also drug interruptions or treatment cessation due to toxicity and lenvatinib-related hospitalisations are a reflection of intolerability.

Serious adverse events were observed in our non-trial population more often than expected from the SELECT study. However, the general safety profile of lenvatinib was comparable to that reported previously, and no new safety concerns were observed $(16,17,18)$.

The high frequency of toxicity is in accordance with other studies $(11,18,19,20,21,22,23)$. Suggestions for improving tolerability include a lower starting dose, combined with dose modifications, in order to improve the safety profile of lenvatinib while maintaining efficacy (21). Dose interruptions are also an option, and improved efficacy was shown in a previous study, regardless of the duration although a shorter interruption was superior in outcome (23). Moreover, previous studies stress the (timely) management of (treatment-emergent) AEs and patient awareness $(19,20)$. Therefore, close monitoring of patients is very important.

Hypertension, the most frequently observed grade $\geq 3$ adverse event, was rather well manageable with antihypertensive agents. Treatment-emerging hypertension has been associated with lenvatinib efficacy (24) and was suggested as a biomarker of TKI efficiency (25). In exploratory post hoc analyses, PFS and OS were estimated for the subgroup of patients who were diagnosed with treatment-emergent hypertension. In this subgroup, median PFS was 16.6 (95\% CI: 11.1-22.0) vs 5.1 months
Table 3 Outcomes in real-life lenvatinib-treated patients. Data are presented as median $(95 \% \mathrm{Cl})$ or as $n(\%)$.

\begin{tabular}{|c|c|c|c|}
\hline \multirow[b]{2}{*}{ Parameters } & \multirow{2}{*}{$\begin{array}{c}\text { Real-life data } \\
\text { All patients } \\
(n=39)\end{array}$} & \multicolumn{2}{|c|}{$\begin{array}{c}\text { SELECT study } \\
\text { (Schlumberger et al. (7)) }\end{array}$} \\
\hline & & $\begin{array}{l}\text { Lenvatinib } \\
(n=261)\end{array}$ & $\begin{array}{l}\text { Placebo } \\
(n=131)\end{array}$ \\
\hline PFS, months & $9.7(4.0-15.5)$ & 18.3 (15.1-NE) & $3.6(2.2-3.7)$ \\
\hline \multicolumn{4}{|l|}{ Rate, \% (95\% Cl) } \\
\hline 6 months & 76 (63-91) & 77.5 (71.7-82.3) & $25.4(18.0-33.6)$ \\
\hline 12 months & 49 (34-70) & $63.0(56.5-68.9)$ & 10.5 (5.7-16.9) \\
\hline 18 months & $36(22-60)$ & $51.1(43.3-58.3)$ & $3.8(1.1-9.2)$ \\
\hline 24 months & $20(9-48)$ & $44.3(35.1-53.1)$ & NE \\
\hline OS, months & $18.3(4.9-31.7)$ & NE (22.0-NE) & NE (14.3-NE) \\
\hline \multicolumn{4}{|l|}{ Rate, \% (95\% Cl) } \\
\hline 6 months & 81 (69-95) & 90.7 (86.4-93.7) & 85.3 (78.0-90.4) \\
\hline 12 months & 64 (49-83) & $81.6(76.2-85.8)$ & $70.0(57.1-79.7)$ \\
\hline 18 months & $54(38-77)$ & 72.3 (65.7-77.9) & $63.0(44.3-76.9)$ \\
\hline 24 months & 49 (33-73) & $58.2(46.0-68.6)$ & NE \\
\hline $\mathrm{RR}^{*}, n(\%)$ & $15(38)$ & 169 (64.8) & $2(1.5)$ \\
\hline $\mathrm{DCR}^{\dagger}, n(\%)$ & $29(74)$ & 229 (87.7) & $73(55.7)$ \\
\hline \multicolumn{4}{|l|}{$\mathrm{BOR}, n(\%)$} \\
\hline CR & $1(3)$ & $4(1.5)$ & 0 \\
\hline PR & $14(33)$ & 165 (63.2) & $2(1.5)$ \\
\hline SD & $14(37)$ & $60(23.0)$ & $71(54.2)$ \\
\hline PD & $2(7)$ & $18(6.9)$ & $52(39.7)$ \\
\hline NE & $8(21)$ & $14(5.4)$ & $6(4.6)$ \\
\hline
\end{tabular}

* $R R$ calculated as $C R+P R$; $+D C R$ calculated as $C R+P R+S D$. $B O R$, best overall response; $\mathrm{Cl}$, confidence interval; $C R$, complete response; $D C R$, disease control rate; $N E$, not estimable; OS, overall survival; PD, progressive disease; PFS, progression-free survival; PR, partial response; RR, response rate; SD, stable disease.

(95\% CI: 3.8-6.5) and median OS was 30.0 (95\% CI: 6.653.4) vs 10.3 months (95\% CI: 2.3-18.2).

The shorter PFS (median PFS 9.7 months in our non-trial population versus 18.3 months in the lenvatinib-treated patients in the SELECT trial) may be accounted for by several patient-related parameters. Differences in baseline characteristics between patients included in our study and those in SELECT were multiple, including more Hürthle cancer as histologic subtype, high baseline TSH and more patients with bone metastases as compared to the SELECT study population. The presence of bone metastases, as these may occur more often to be RAI-refractory, is known to be associated with poor survival in differentiated thyroid cancer (26). A higher percentage of our patients had received prior lines of TKI therapy; potentially indicative of a worse overall health and a more advanced disease stage of our patients at the start of lenvatinib treatment as compared to the SELECT trial population. Overall, almost three quarter of our population did not comply with the eligibility criteria of the SELECT trial.

Our data regarding PFS, as shown in the forest plot (Fig. 4), and OS were comparable to other real-life studies of lenvatinib $(11,12,13)$. Our data show a median OS of 18.3 months. However, in the SELECT trial median OS 
Schlumberger, 2015

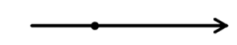

Sugino, 2018

$\bullet$

Berdelou, 2017

Balmelli, 2018

Nervo, 2018

Aydemirli, 2019

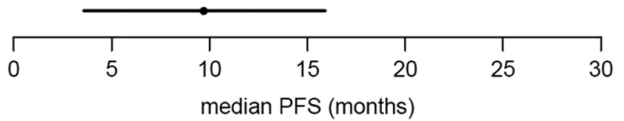

\section{Figure 4}

Forest plot of median PFS under lenvatinib use. Outcomes for median PFS with their (*approximated) $95 \% \mathrm{Cl}$ across various lenvatinib real-life studies (Berdelou et al.*, Balmelli et al., Nervo et al., our study) and trials (Schlumberger et al., Sugino et al.) are visualised. For the study of Sugino et al., 95\% Cl was not reported and could not be obtained from the information presented in the article. References in main text. Arrowheads indicate that the upper bound of the $95 \%$ confidence interval was not estimable.

was not established; cumulative survival up to 20 months was approximately 70\% (7). Data on median OS for lenvatinib in thyroid cancer is scarce and reported in two real-life studies only, with small patient groups of 12 and 13 patients respectively $(12,13)$. However, OS in these studies was comparable to our data.

Limitations include the retrospective nature of our data acquisition, which may have been performed or documented less stringently than in a trial population, partially leading to the larger proportion of missing evaluations. Furthermore, toxicity has been scored retrospectively from the electronic health records. The sample size of our real-life study may be too small to draw very precise conclusions and multivariable analyses were decided not to be performed. However, nearly all patients who have been treated in the Netherlands with lenvatinib until January 2019 for thyroid cancer have been included in this study, which enables us to evaluate this treatment option in daily practice.

Our study shows that outcomes in real-life significantly differ from the results from clinical trials, on which approval of the drug-regulating authorities is based. Eligibility criteria are more stringently applied in clinical studies. However, in clinical practice therapeutic alternatives are not always available and less fit patients with comorbidities might choose to start treatment despite a higher risk of toxicity. It seems inherent to the disease course, that factors such as not having undergone standard therapy (i.e. due to an initial presentation with metastatic disease, thus an already advanced stage disease and poor prognosis) is found to be associated with worse outcome. However, also these patients are part of the real-life population and in clinical practice may be offered treatment with lenvatinib. Detailed real-life data may aid in the process of shared decision making in the treatment of thyroid cancer.

Furthermore, we consider that drug efficacy may be potentially overestimated in the clinical trial, if certain patients are selected for rendition of the PFS. For instance, selecting patients based on absence of the emergence of toxicity could potentially lead to an overestimation of PFS. It is not evident if toxicity-related censoring has occurred in the calculation of the median PFS in the article by Schlumberger et al. (7) and Gianoukakis et al. (10) when in the latter the PFS curve is designed as appertaining the lenvatinib responders.

\section{Conclusions}

Our data show that treatment with lenvatinib in reallife RR-DTC patients in the Netherlands is effective and comparable to that of other real-life studies, although PFS is significantly shorter than in the SELECT trial. Lenvatinib showed a serious general toxicity profile comparable to that reported in the SELECT study population. In daily clinical practice, overall tolerability seems feasible in a subset of patients, with timely management of adverse events and patient awareness, dose adjustments or temporary interruptions.

\section{Declaration of interest}

E K: Advisory boards BMS, Novartis, Roche, Merck, Amgen, Pierre-Fabre, EISAI, Bayer, Genzyme-Sanofi. Research grants from Novartis and BMS. T P L: Advisory boards EISAl, Bayer, Genzyme-Sanofi. R G declares no conflicts of interest. R G was financially supported by grants from the Netherlands Organization for Scientific Research (ZonMW-Vidi project 917.16.430) and the LUMC, Leiden, the Netherlands. The other authors have nothing to disclose.

\section{Funding}

This research did not receive any specific grant from any funding agency in the public, commercial or not-for profit sector. 


\section{References}

1 Lim H, Devesa SS, Sosa JA, Check D \& Kitahara CM. Trends in thyroid cancer incidence and mortality in the United States, 1974-2013. JAMA 2017317 1338-1348. (https://doi.org/10.1001/jama.2017.2719)

2 Lloyd RV, Osamura RY, Klöppel G \& Rosai J. WHO Classification of Tumours of Endocrine Organs, Vol. 10, 4th ed. Lyon: International Agency for Research on Cancer, 2017.

3 Haugen BR, Alexander EK, Bible KC, Doherty GM, Mandel SJ, Nikiforov YE, Pacini F, Randolph GW, Sawka AM, Schlumberger M et al. 2015 American Thyroid Association management guidelines for adult patients with thyroid nodules and differentiated thyroid cancer: the American Thyroid Association Guidelines Task Force on thyroid nodules and differentiated thyroid cancer. Thyroid 201626 1-133. (https://doi.org/10.1089/thy.2015.0020)

4 Schlumberger M, Brose M, Elisei R, Leboulleux S, Luster M, Pitoia F \& Pacini F. Definition and management of radioactive iodine-refractory differentiated thyroid cancer. Lancet: Diabetes and Endocrinology 2014 2 356-358. (https://doi.org/10.1016/S2213-8587(13)70215-8)

5 Durante C, Haddy N, Baudin E, Leboulleux S, Hartl D, Travagli JP, Caillou B, Ricard M, Lumbroso JD, De Vathaire F et al. Long-term outcome of 444 patients with distant metastases from papillary and follicular thyroid carcinoma: benefits and limits of radioiodine therapy. Journal of Clinical Endocrinology and Metabolism 200691 2892-2899. (https://doi.org/10.1210/jc.2005-2838)

6 Brose MS, Smit J, Capdevila J, Elisei R, Nutting C, Pitoia F, Robinson B, Schlumberger M, Shong YK \& Takami H. Regional approaches to the management of patients with advanced, radioactive iodine-refractory differentiated thyroid carcinoma. Expert Review of Anticancer Therapy 201212 1137-1147. (https://doi. org/10.1586/era.12.96)

7 Schlumberger M, Tahara M, Wirth LJ, Robinson B, Brose MS, Elisei R, Habra MA, Newbold K, Shah MH, Hoff AO et al. Lenvatinib versus placebo in radioiodine-refractory thyroid cancer. New England Journal of Medicine 2015372 621-630. (https://doi.org/10.1056/ NEJMoa1406470)

8 Agrawal VR, Jodon G, Mushtag R \& Bowles DW. Update on multikinase inhibitor therapy for differentiated thyroid cancer. Drugs of Today 201854 535-545. (https://doi.org/10.1358/ dot.2018.54.9.2878150)

9 Zschäbitz S \& Grüllich C. Lenvantinib: a tyrosine kinase inhibitor of VEGFR 1-3, FGFR 1-4, PDGFRalpha, KIT and RET. Recent Results in Cancer Research 2018211 187-198.

10 Gianoukakis AG, Dutcus CE, Batty N, Guo M \& Baig M. Prolonged duration of response in lenvatinib responders with thyroid cancer. Endocrine-Related Cancer 201825 699-704. (https://doi.org/10.1530/ ERC-18-0049)

11 Berdelou A, Borget I, Godbert Y, Nguyen T, Garcia ME, Chougnet CN, Ferru A, Buffet C, Chabre O, Huillard O et al. Lenvatinib for the treatment of radioiodine-refractory thyroid cancer in real-life practice. Thyroid 201728 72-78. (https://doi.org/10.1089/thy.2017.0205)

12 Balmelli C, Railic N, Siano M, Feuerlein K, Cathomas R, Cristina V, Guthner C, Zimmerman S, Weidner S, Pless $\mathrm{M}$ et al. Lenvatinib in advanced radioiodine-refractory thyroid cancer - a retrospective analysis of the Swiss lenvatinib named patient program. Journal of Cancer 20189 250-255. (https://doi.org/10.7150/jca.22318)

13 Nervo A, Gallo M, Sama MT, Felicetti F, Alfano M, Migliore E, Marchisio F, Berardelli R, Arvat E \& Piovesan A. Lenvatinib in advanced radioiodine-refractory thyroid cancer: a snapshot of reallife clinical practice. Anticancer Research 201838 1643-1649. (https:// doi.org/10.21873/anticanres.12396)
14 Sugino K, Nagahama M, Kitagawa W, Ohkuwa K, Uruno T, Matsuzu K, Suzuki A, Masaki C, Akaishi J, Hames KY et al. Clinical factors related to the efficacy of tyrosine kinase inhibitor therapy in radioactive iodine refractory recurrent differentiated thyroid cancer patients. Endocrine Journal 201865 299-306. (https://doi. org/10.1507/endocrj.EJ17-0365)

15 Simon R. Confidence intervals for reporting results of clinical trials. Annals of Internal Medicine 1986105 429-435. (https://doi. org/10.7326/0003-4819-105-3-429)

16 Berdelou A, Lamartina L, Klain M, Leboulleux S, Schlumberger $\&$ TUTHTYREF Network. Treatment of refractory thyroid cancer. Endocrine-Related Cancer 201825 209-223.

17 Yu ST, Ge JN, Luo JY, Wei ZG, Sun BH \& Lei ST. Treatment-related adverse effects with TKIs in patients with advanced or radioiodine refractory differentiated thyroid carcinoma: a systematic review and meta-analysis. Cancer Management and Research 201911 1525-1532. (https://doi.org/10.2147/CMAR.S191499)

18 Hayato S, Shumaker R, Ferry J, Binder T, Dutcus CE \& Hussein Z. Exposure-response analysis and simulation of lenvatinib safety and efficacy in patients with radioiodine-refractory differentiated thyroid cancer. Cancer Chemotherapy and Pharmacology 201882 971-978. (https://doi.org/10.1007/s00280-018-3687-4)

19 Cabanillas ME \& Takahashi S. Managing the adverse events associated with lenvatinib therapy in radioiodine-refractory differentiated thyroid cancer. Seminars in Oncology 201946 57-64. (https://doi.org/10.1053/j.seminoncol.2018.11.004)

20 Resteghini C, Cavalieri S, Galbiati D, Granata R, Alfieri S, Bergamini C, Bossi P, Licitra L \& Locati LD. Management of tyrosine kinase inhibitors (TKI) side effects in differentiated and medullary thyroid cancer patients. Best Practice and Research: Clinical Endocrinology and Metabolism 201731 349-361. (https://doi. org/10.1016/j.beem.2017.04.012)

21 Yamazaki H, Iwasaki H, Takasaki H, Suganuma N, Sakai R, Masudo K, Nakayama H, Rino Y \& Masuda M. Efficacy and tolerability of initial low-dose lenvatinib to treat differentiated thyroid cancer. Medicine 201998 e14774. (https://doi.org/10.1097/ MD.0000000000014774)

22 Capdevila J, Newbold K, Licitra L, Popovtzer A, Moreso F, Zamorano J, Kreissl M, Aller J \& Grande E. Optimisation of treatment with lenvatinib in radioactive iodine-refractory differentiated thyroid cancer. Cancer Treatment Reviews 201869 164-176. (https://doi. org/10.1016/j.ctrv.2018.06.019)

23 Tahara M, Brose MS, Wirth LJ, Suzuki T, Miyagishi H, Fujino K, Dutcus CE \& Gianoukakis A. Impact of dose interruption on the efficacy of lenvatinib in a phase 3 study in patients with radioiodinerefractory differentiated thyroid cancer. European Journal of Cancer 2019106 61-68. (https://doi.org/10.1016/j.ejca.2018.10.002)

24 Wirth LJ, Tahara M, Robinson B, Francis S, Brose MS, Habra MA, Newbold K, Kiyota N, Dutcus CE, Mathias E et al. Treatmentemergent hypertension and efficacy in the phase 3 study of (E7080) lenvatinib in differentiated cancer of the thyroid (SELECT). Cancer 2018124 2365-2372. (https://doi.org/10.1002/cncr.31344)

25 Budolfsen C, Faber J, Grimm D, Kruger M, Bauer J, Wehland M, Infanger M \& Magnusson NE. Tyrosine kinase inhibitor-induced hypertension: role of hypertension as a biomarker in cancer treatment. Current Vascular Pharmacology 201917 618-634. (https:// doi.org/10.2174/1570161117666190130165810)

26 Califano I, Deutsch S, Lowenstein A, Cabezon C \& Pitoia F. Outcomes of patients with bone metastases from differentiated thyroid cancer. Archives of Endocrinology and Metabolism 201862 14-20. (https://doi. org/10.20945/2359-3997000000004)

Received 25 September 2019

Revised version received 11 November 2019

Accepted 14 November 2019 